\title{
Allergic Bronchopulmonary Aspergillosis: A Diagnostic Challenge
}

Saraswati Pokharel ${ }^{*}$, Lourdes Ylagan and Richard Cheney

Department of Pathology and Laboratory Medicine, Roswell Park Cancer Institute, USA

*Corresponding author: Saraswati Pokharel, Roswell Park Cancer Institute, Elm and Carlton St, Buffalo, NY 14263, USA, Tel: 7168454959 ; Fax: 716 845 2370; Email: saraswati.pokharel@roswellpark.org

Rec date: Oct 23, 2014, Acc date: Nov 28, 2014, Pub date: Dec 01, 2014

Copyright: $\odot 2014$ Pokharel S, et al. This is an open-access article distributed under the terms of the Creative Commons Attribution License, which permits unrestricted use, distribution, and reproduction in any medium, provided the original author and source are credited.

\begin{abstract}
Allergic bronchopulmonary aspergillosis (ABPA) is an immunologic condition that results from an allergic immune response to Aspergillus fumigatus, most often occurring in a patient with bronchial asthma or cystic fibrosis. ABPA is diagnosed by constellation of clinical, laboratory, and radiographic criteria. In the absence of typical presentation, ABPA can be misdiagnosed. Our patient presented with a $3 \mathrm{~cm}$ right lower lobe lung mass and sub-centimeter right upper lobe lung nodules. The clinical features led to a presumptive diagnosis of lung carcinoma. The patient underwent preoperative bronchial washing and endobronchial biopsy. The washing sample showed large amount of thick mucus containing abundant eosinophils, Charcoat-Leyden crystals, and degenerated cellular debris consistent with "allergic mucin". These findings were initially overlooked and considered non-specific. Repeat sampling (needle biopsy) showed marked reactive pneumocyte hyperplasia in the background of inflammation, which was misdiagnosed as adenocarcinoma with lepidic growth pattern. The correct diagnosis was made only after the surgical resection of the lesion. Diagnosis of ABPA can be missed due to general unfamiliarity with this entity and its clinical presentation similar to the lung tumor. Accurate diagnosis can be derived from the bronchial washing if the features of "allergic mucin" are recognized and confirmed with microbiological examination.
\end{abstract}

Keywords: Bronchopulmonary aspergillosis; Allergy; Mucin; Aspergillus; Lung

\section{Introduction}

ABPA is an allergic pulmonary disorder usually caused by hypersensitivity to Aspergillus fumigatus (A fumigatus) that manifests with combination of clinical, laboratory, and radiographic findings including chronic asthma, recurrent pulmonary infiltrates, bronchiectasis, serum eosinophilia, an elevated total IgE level, and sensitization to $A$ fumigatus by skin testing [1-3]. Occasionally, patients can develop a syndrome similar to ABPA caused by fungi other than $A$ fumigatus and is called allergic bronchopulmonary mycosis [4]. The clinical and radiological features of ABPA are extensively reported in the literature. To our knowledge, the cytological findings associated with ABPA, particularly in the context of specimen obtained from bronchial washing, has not been reported. Here, we report a case of ABPA with unusual clinical and radiological features that was misdiagnosed as lung carcinoma during preoperative workup.

\section{Case Report}

A 76-year-old male in general good health complained of progressive cough over the past year, which at times brought up large brown specks. He did not carry the diagnosis of bronchial asthma or cystic fibrosis. He was treated with courses of antibiotics that offered temporary relief. In the meantime, patient also developed abdominal pain and rectal bleeding for which he underwent computed tomography (CT) scan abdomen that showed right lower lobe (RLL) mass. Further workup with CT chest confirmed a $3.6 \times 2.3 \times 2.2 \mathrm{~cm}$ lobulated RLL mass and a $6 \mathrm{~mm}$ right upper lobe nodule. He underwent a bronchoscopy with bronchial washing and transbronchial biopsy of the lesion. Biopsy showed non-specific changes including reactive pneumocyte hyperplasia associated with chronic inflammation including rare interstitial eosinophils. Patient was then referred to our institution for further clinical work up and management. Repeat CT scan after a month showed a stable dominant $3 \times 2 \mathrm{~cm}$ RLL mass with enlarging satellite nodules (Figure 1). Also noted were stable subcentimeter nodules in both lungs. A PET scan showed uptake in the RLL mass and a right hilar lymph node.

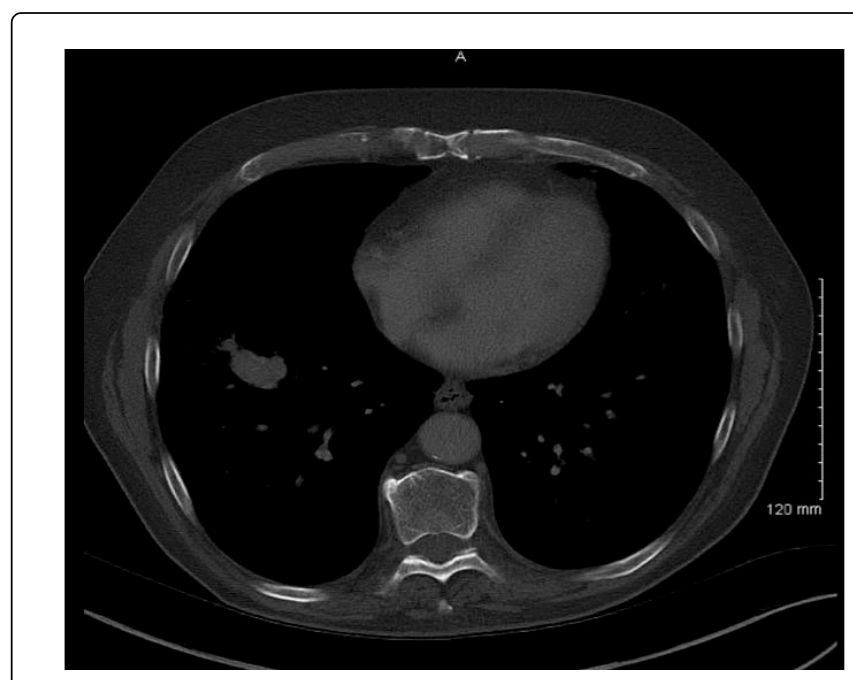

Figure 1: CT chest showing $3 \mathrm{~cm}$ rigth lower lobe lobulated mass.

Subsequently, patient underwent a percutaneous biopsy of the lower lobe mass that showed reactive pneumocyte hyperplasia with atypia in a background of acute and chronic inflammation. These reactive pneumocytes were mistaken for adenocarcinoma with lepidic growth pattern. The patient eventually underwent a video assisted 
right upper lobe wedge resection, right lower lobectomy as well as mediastinoscopy with lymph node sampling. Right upper lobe wedge contained two firm, ill-defined nodules, each measuring $0.5 \mathrm{~cm}$ in greatest dimension. Microscopic examination of the nodules showed poorly-formed non-necrotizing granulomas in the background of organizing pneumonia. Similarly, a $4.5 \times 3.4 \times 2 \mathrm{~cm}$, firm tan mass with a focally friable cut surface was identified on the right lower lobectomy. The bronchi and bronchioles appeared dilated with soft, $\tan$ mucoid material in the lumen. Microscopic examination of the sections taken from the mass showed markedly dilated proximal airways with lumens obliterated by thick mucous plugs and destruction of airway wall with marked chronic inflammation. These mucus plugs were composed of abundant intact and degenerated eosinophils, Charcot-Leyden crystals, degenerated respiratory cells and fungal hyphae. The fungal hyphae were identified with Hematoxylin and Eosin (H\&E) staining as well as Gomori methenamine silver (GMS) staining. The surrounding lung parenchyma showed variable histologic changes including intraalveolar fibrin deposits, interstitial and intra-alveolar eosinophils, chronic inflammatory cells, and multifocal, myxoid fibrous tissue obliterating the alveolar spaces. These findings were consistent with acute eosinophilic pneumonia with focal organization (Figure 2). One of the peribronchial lymph nodes also showed a focus of hyalinized granuloma. The constellation of findings is consistent with ABPA.

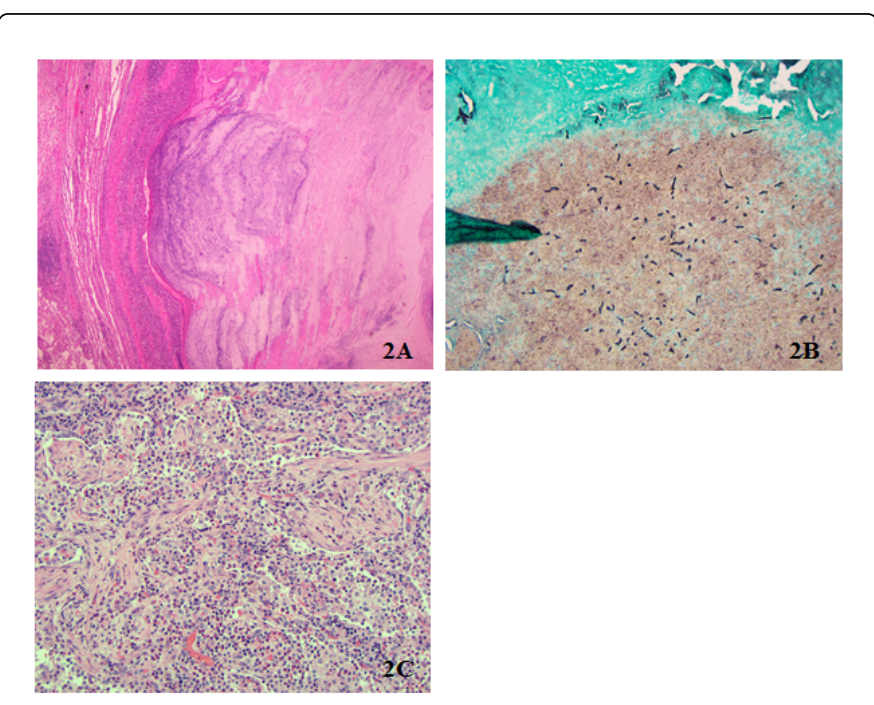

Figure 2: Histology images of the sections from lung mass. A. Allergic mucin within the lumen of the dilated airways, Hematoxylin and Eosin stain, 40X magnification; B. Fungal hyphae highlighted by GMS stain; and C. Surrounding lung parenchyma with intraalveolar eosinophils and organizing pneumonia, Hematoxylin and Eosin stain, 200X magnification.

Once the diagnosis was established, the tissue block of the previous bronchial washing was obtained from the outside institution and rereviewed. Microscopic examination of the H\&E stained section showed abundant eosinophils, Charcot-Leyden crystals and degenerated respiratory cells admixed with mucin. The cytomorphology of this specimen was identical to the "allergic mucin" present in the lumen of the dilated proximal airways in the lower lobe lobectomy specimen. While fungal elements were not readily identified on H\&E stain, GMS stain highlighted few fragmented fungal hyphae (Figure 3). Since undergoing lung resection, the patient's cough significantly improved and he is doing well.

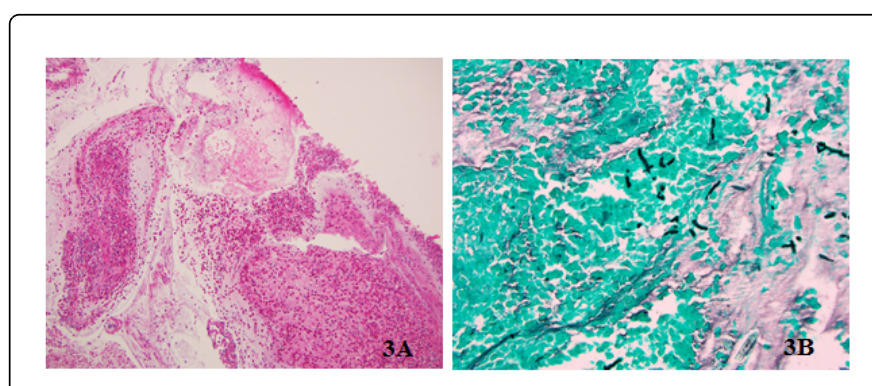

Figure 3: Cell block sections of bronchial washing fluid. (A) Numerous viable and degenerated eosinophils, Charcot-Leyden crystals and thick mucin. Hematoxylin and Eosin stain, $100 \mathrm{X}$ magnification; (B) Fungal hyphae highlighted by GMS stain.

\section{Discussion}

This patient presented with signs, symptoms and radiologic findings highly suggestive of lung carcinoma. Except for a short period of progressive cough, the patient did not have any other history of chronic lung diseases including bronchial asthma or cystic fibrosis. When referred for bronchoscopic examination, the usual findings of ABPA such as central bronchiectasis, thick mucus plug and inflamed airway wall were not identified. The findings on transbronchial biopsy were non-specific but important cytomorphologic features present in the bronchial washing were not overlooked.

ABPA may have no typical radiologic manifestations, but usually several abnormalities are present. Central bronchiectasis, which is considered a specific finding, may be obvious, subtle, or absent. Other findings may include hyperinflation, varying infiltrates or areas of consolidation, nodules, and manifestations of bronchiectasis, including linear opacities, ring opacities, and gloved-finger opacities representing mucoid impaction [3,5]. In this patient, CT chest demonstrated dominant $3 \mathrm{~cm}$ mass. However, other features such as central bronchiectasis and bronchial wall thickening, which characteristically accompanies ABPA [6], were not noticed.

Typically, patients with ABPA have history of bronchial asthma or cystic fibrosis [3]. The susceptibility of some individuals, particularly cystic fibrosis and asthmatic patients to develop ABPA is not fully understood. Some reports suggest that exposure to large concentrations of spores of $A$ fumigatus may cause ABPA $[7,8]$. However, environmental factors are not considered to be the main pathogenic factors because not all asthmatics develop ABPA despite being exposed to the same environment. In a genetically predisposed individual [9,10], inhaled conidia of $A$ fumigatus persist and germinate into hyphae with release of antigens that compromise the mucociliary clearance, stimulate and breach the airway epithelial barrier, and activate the innate immunity of the lung [11,12].

The pathology of ABPA varies from patient to patient, and in different areas of the lung in the same patient. Histologic examination reveals mostly an airway centered disease process characterized by dilated airways filled with mucus containing large number of inflammatory cells, predominantly eosinophils, and Charcot-Leyden crystals. Fungal hyphae can often be demonstrated in the bronchiectatic cavities. The bronchial wall in ABPA is usually 
infiltrated by mixed inflammatory cells, primarily eosinophils. The peribronchial parenchyma shows an inflammatory response with conspicuous eosinophilia and intraalveolar fibrin, a pattern similar to that of eosinophilic pneumonia. Long standing cases may show features of organizing pneumonia [13]. Bronchocentric granulomatosis, the presence of noncaseating granulomas containing palisaded histiocytes and multinucleated giant cells centered on the airway, are also seen [14]. A majority of the typical histological findings were identified in the resection specimen of this patient. More importantly, bronchial washing demonstrated large amount of mucin, exfoliated respiratory cells, and inflammatory cells including significant number of eosinophils and Charcot-Leyden crystals. This is similar to the allergic mucin described in fungal sinusitis, a condition occasionally associated with ABPA.

Conclusion: $\mathrm{ABPA}$ is a relatively rare entity that creates a diagnostic difficulty in the absence of typical clinical and radiological presentation. In some cases chest CT scan may indicate a diagnosis of lung cancer, and biopsy may show pneumocyte atypia, acute and chronic inflammation but not frankly malignant cells. In these circumstances simulating a lung tumor the physicians should consider ABPA, and cytological findings of numerous eosinophils in the bronchial washing could prompt further microbiological tests to improve the differential diagnosis and avoid unnecessary surgery.

\section{References}

1. Greenberger PA (2002) Allergic bronchopulmonary aspergillosis. J Allergy Clin Immunol 110: 685-692.

2. Rosenberg M, Patterson R, Mintzer R, Cooper BJ, Roberts M, et al. (1977) Clinical and immunologic criteria for the diagnosis of allergic bronchopulmonary aspergillosis. Ann Intern Med 86: 405-414.

3. Greenberger PA (2013) When to suspect and work up allergic bronchopulmonary aspergillosis. Ann Allergy Asthma Immunol 111: 1-4.
4. Muscat I, Oxborrow S, Siddorn J (1988) Allergic bronchopulmonary mycosis. Lancet 1: 1341 .

5. Patterson R, Greenberger PA, Halwig JM, Liotta JL, Roberts M (1986) Allergic bronchopulmonary aspergillosis. Natural history and classification of early disease by serologic and roentgenographic studies. Arch Intern Med 146: 916-918.

6. [No authors listed] (2001) Case records of the Massachusetts General Hospital. Weekly clinicopathological exercises. Case 24-2001. A 46-yearold woman with chronic sinsusitis, pulmonary nodules, and hemoptysis. N Engl J Med 345: 443-449.

7. Henderson AH (1986) Allergic aspergillosis: review of 32 cases. Thorax 23:513-523

8. Allmers H, Huber H, Baur X (2000) Two year follow-up of a garbage collector with allergic bronchopulmonary aspergillosis (ABPA). Am J Ind Med 37: 438-442.

9. Chauhan B, Santiago L, Hutcheson PS, Schwartz HJ, Spitznagel E, et al. (2000) Evidence for the involvement of two different MHC class II regions in susceptibility or protection in allergic bronchopulmonary aspergillosis. J Allergy Clin Immunol 106: 723-729.

10. Eaton TE, Weiner Miller P, Garrett JE, Cutting GR (2002). Cystic fibrosis transmembrane conductance regulator gene mutations: do they play a role in the aetiology of allergic bronchopulmonary aspergillosis? Clin Exp Allergy 32: 756-761.

11. Hogaboam CM, Blease K, Schuh JM (2003) Cytokines and chemokines in allergic bronchopulmonary aspergillosis (ABPA) and experimental Aspergillus-induced allergic airway or asthmatic disease. Front Biosci 8: e147-156.

12. Kauffman HF (2003) Immunopathogenesis of allergic bronchopulmonary aspergillosis and airway remodeling. Front Biosci 8: e190-196.

13. Slavin RG, Bedrossian CW, Hutcheson PS, Pittman S, Salinas-Madrigal L, et al. (1988) A pathologic study of allergic bronchopulmonary aspergillosis. J Allergy Clin Immunol 81: 718-725.

14. Kradin RL, Mark EJ (2008) The pathology of pulmonary disorders due to Aspergillus spp. Arch Pathol Lab Med 132: 606-614. 